Article

\title{
Time Optimization of Unmanned Aerial Vehicles Using an Augmented Path
}

\author{
Abdul Quadir Md ${ }^{1}$, Divyank Agrawal ${ }^{1}$, Monark Mehta ${ }^{1}$, Arun Kumar Sivaraman ${ }^{1}$ (I) and Kong Fah Tee ${ }^{2, *(D)}$ \\ 1 Vellore Institute of Technology (VIT), School of Computer Science and Engineering, Chennai 632002, India; \\ abdulquadir.md@vit.ac.in (A.Q.M.); divyank.agrawal2019@vitstudent.ac.in (D.A.); \\ monark.mehta2019@vitstudent.ac.in (M.M.); arunkumar.sivaraman@vit.ac.in (A.K.S.) \\ 2 School of Engineering, University of Greenwich, Kent ME4 4TB, UK \\ * Correspondence: K.F.Tee@gre.ac.uk
}

check for updates

Citation: Md, A.Q.; Agrawal, D.; Mehta, M.; Sivaraman, A.K.; Tee, K.F. Time Optimization of Unmanned Aerial Vehicles Using an Augmented Path. Future Internet 2021, 13, 308. https://doi.org/10.3390/fi13120308

Academic Editors: Xavier Fernando and Kandasamy Illanko

Received: 31 October 2021

Accepted: 28 November 2021

Published: 29 November 2021

Publisher's Note: MDPI stays neutral with regard to jurisdictional claims in published maps and institutional affiliations.

Copyright: (c) 2021 by the authors. Licensee MDPI, Basel, Switzerland. This article is an open access article distributed under the terms and conditions of the Creative Commons Attribution (CC BY) license (https:/ / creativecommons.org/licenses/by/ $4.0 /)$.

\begin{abstract}
With the pandemic gripping the entire humanity and with uncertainty hovering like a black cloud over all our future sustainability and growth, it became almost apparent that though the development and advancement are at their peak, we are still not ready for the worst. New and better solutions need to be applied so that we will be capable of fighting these conditions. One such prospect is delivery, where everything has to be changed, and each parcel, which was passed people to people, department to department, has to be made contactless throughout with as little error as possible. Thus, the prospect of drone delivery and its importance came around with optimization of the existing system for making it useful in the prospects of delivery of important items like medicines, vaccines, etc. These modular AI-guided drones are faster, efficient, less expensive, and less power-consuming than the actual delivery.
\end{abstract}

Keywords: pandemic; modular; AI; delivery system; drones; unmanned aerial vehicle

\section{Introduction}

In today's world, technology is getting increasingly more advanced and complex with every moment passing by. People are finding more ways to utilize energy in a more efficient way to obtain maximum output out of a machine, i.e., increasing efficiency to obtain the most use out of technology. Moreover, there is also the development of new technologies to boost the advancement and reduce human effort, minimize error, and bring about change for the betterment of society and mankind.

About 100 years back, when two brothers left the land and took the first step towards the sky, mankind has not looked back since then. Rather, they are striving further away from land towards the sky and universe. Since then, there have been multiple advancements and now the sky is within our grasp. Still, it is difficult for many in the world to access these wonders due to their large size, cost, etc. Now, these problems have also been fixed as they are cheaper and can fit in the palm of our hand and are available everywhere. Moreover, like every other technology, for the next step, they are now being used for daily aspects of our life. These small highly efficient systems are unmanned aerial vehicles (UAVs).

This paper is about increasing the efficiency so that they can perform better in that aspect of life. For example, recently, there is need for advancement in the delivery system, as the world is going through the COVID-19 pandemic, which is transmitted through human contact. There was a sudden need for contactless delivery and so drone trials were conducted for these deliveries, and now are being improved to completely take over human delivery. There is a major shortcoming in this area of research. While it is being advanced to follow the instructions to have a successful delivery, it is still running on a GPS and land tracking system. Now, these systems have the basic idea and outcome to give the minimum distance between two points, which is true in the case of travelling by land as all the constraints like mileage, performance, efficiency, etc. are in terms of distance 
and speed. For instance, during a journey by land, it is generally described through two points or by distance like Mumbai to Pune, but in aviation, the journey is by time like a 2-hour flight, so clearly, time is of greater priority.

On the other hand, UAVs are aerial vehicles where time is the measure of efficiency and output. A drone's efficiency is calculated by how much flight time is given by it. Thus, GPS (used for coordinates or tracking) cannot be used as a path. The more efficient path is one using less time. Moreover, with the drone's tech, distance and displacement mean the same thing. The upthrust and the forward speed work in sync to move the drone while the main challenge is to follow a certain path.

The primary challenges discussed in this paper are as follows:

- First, the drone has to follow the path. For this, the path should be defined and also be a system that controls the motors and the body of the UAVs for the path to follow;

- Secondly, the height limits. There are certain maximum heights for UAVs to fly at, which are decided by the legal bodies. It limits the area and also the path choices and the efficiency as there has to be a constant path where height would not increase though the UAVs still move forward;

- The UAVs are automatic. The machine should be capable of making the decisions of when to start, ascend or descend, or just move straight;

- As there are no guides or drivers, the machine should be self-driven. The air is full of traffic both natural and man-made, with buildings also soaring beyond imagination into the sky. The UAVs must be installed with an AI or guiding system to overcome barriers, precautions, and damage control, and also specific conditions as to when to activate what system. This must be overlooked by an AI, which is complex to develop;

- As the UAV is automatic and its development will result in an increase, there must be some tracking systems that continuously transmit and store ion servers with all the changes during the journey until the journey is completed and during an emergency, it should be located easily.

In this paper, we design an algorithm that will not only define the path of the drone to reach its destination, but will also be an automated path, i.e., it does not require piloting, rather it is self-driven, and this algorithm helps to increase the efficiency and provide higher output and thus it can carry out a more complex task at a greater speed.

\section{Literature Survey}

The delivery of products using a drone is not very popular in a country with huge diversity like India. We are working on an efficient algorithm to reduce the time efficiency of the drone so that the drone can deliver the product by saving time [1]. Research has investigated the optimization of modular drones so that they can form an efficient delivery system. Specifically, the battery life and motor have been investigated to save time and prevent delays in the delivery of products. The carrier placed in the drone should be strong enough has also been considered so that it can handle the weight of the parcel [2]. A study has also been performed considering the dense traffic. They developed the shortest path to deliver the product to the customer, and also stated that they have to install a recharging system in a different location to recharge the drone when needed so that there will be no delay due to a power deficiency [3]. Cost-efficient delivery of their product has also been explored by making an algorithm to solve the vehicle routing problem, which is to deliver to as many customers as possible in a particular set, which in turn saves the cost of drones, fuels, etc. [4]. The efficient delivery of parcels has also been researched by considering the vehicle routing problem, which is the major problem, but this is solved by using some mathematical equations and graphs so that it is easy to deliver in a particular set. They have thought of equipping drones in a particular vehicle and the vehicle stands in the middle of all the locations and the drones when all the parcels are delivered in a particular time, which saves a lot of time and it a cost-efficient idea [5]. Research has been pereformed on battery-efficient drones' delivery, which makes the drones work more efficiently and can provide more working hours for the delivery of parcels. He decided 
to insert two batteries in the drone that are fully charged and if one of the batteries is discharged, then the other battery replaces the position of the battery, i.e., the two batteries are swapped, thus making it more efficient [6-8]. An algorithm has been developed for energy and range minimization, which will consequently help in cost-minimizing. This will also make their delivery very easy and efficient. They have considered a vehicle that runs in the delivery place and finds the shortest part for every nearby location by using some mathematical graphs and algorithms and from the location, the vehicle will launch a drone, which delivers to all the nearby places and returns to the vehicle before the battery is discharged. This idea will make delivery to all the nearby parcels very easy. All these studies mostly worked on the shortest path, most efficient battery, and fastest delivery, but no research has worked on efficient delivery based on the wind flow and environment to save time for the delivery [9-13]. The drone delivery is air transport, but in air transport, the basic constraint for the measurement of efficiency is flight time. All air transport is calculated in terms of flight time and so the basic idea and thus the tracking system is useless. As such, the shortest distance might not give the shortest time. This research paper is about this aspect [14-17], mainly how to reduce time by using basic physics and algorithms to follow that path so that less time is used for reaching the destination and even considering the external interferences like wind speed, height, thrust, and even the weight of the carriage [18-20]. This paper proposes fuzzy control using two combinations of the algorithm: Virtual Reference Feedback Tuning (VRFT) and Active Disturbances Rejection Control (ADRC), to obtain the benefits of data-driven control and fuzzy control. This algorithm saves time in finding the optimal parameters of the controllers [21]. Working on the control problem for stochastic nonlinear systems, it is also based on fuzzy logic systems. They have also considered it with unmeasured states and unknown backlashlike hysteresis, and this is done to reduce the communication load over the system [22]. They have studied the concept of digital twins for an internal transport system, which is becoming more popular nowadays. It supports technologies like material flow. This system increases the efficiency of the production system [23]. A cyber-attack risk analysis that integrates Kaplan's and Garrick's approach and fuzzy theory is proposed, focusing on some of the main targets (database, internal networks, machinery) to prevent cyber attacks. This approach can be used for any type of mining and the obtained result will reveal the current cybersecurity status [24].

\section{Problem Statement}

In air transport, the basic constraint for the measurement of efficiency is flight time. All air transport is calculated in terms of the flight time and so the basic GPS and thus the tracking system is useless, as the shortest distance might not give the shortest time. This research paper concerns this aspect [25-28].

The UAVs need a new set of instructions as they are self-driven and the algorithm has to feed in the coordinates in real-time to the UAVs to keep the drone on track and keep it from deviating from its path. Additionally, it has to check the coordinates for the destination and mid-point of the journey to change its modes. Moreover, the natural terrain and the legal boundaries have to be kept in mind. There is also a set of precautionary or emergency instructions too in case of any failure or damage. The UAVs should also be sending this information to a server or storing it to keep track and recover in case of system failure.

How to reduce time by using basic physics and algorithms to follow that path so that less time is used for reaching the destination and even considering the external interferences like wind speed, height, thrust, and even the weight of the carriage are investigated.

\section{Proposed Augmented Path-Time Architecture}

In this paper, we found that there is a major flaw in UAV technology. Although the software is efficient, it is based on distance optimization techniques on which all the other 
land transportation is based. Rather, the UAVs or other aerial vehicles have time as a major factor for calculating their efficiency and output.

We used some great theories of physics and implemented them on aerial transportation to decrease the time and increase the output.

In modern chipsets like mamba f7 above, they have started to add and modify the flight controllers to the needs of the users. Not only do they have new features like Wi-Fi, Bluetooth, and GPS tracking device, but they also provide a whole set of different empty sockets and even storage and chips to modify, integrate, and even change the functioning of the flight controller to our needs. Although it is still in development, most modern flight controllers have this functionality, making it expensive and complex.

\subsection{Blackbox}

It contains $16 \mathrm{~m}$ flash memory and thus the processing and temporary storage of the algorithm. All the cache and real-time applications run in this part. It is the brain of the quad that carries out all the functionality of the algorithm and gives out the coordinates in real-time. It controls the quad and calls for the values to generate the required results to carry out the task.

\subsection{Gyro Meter}

It is the main component of the flight controller as it calculates the roll, yaw, and pitch and keeps the balance of the UAVs, and keeps it steady. Additionally, it has some onboard memory for storing and modifying purposes. The gyro meter keeps checking the angle of the inclination of the drone as well as the pitch and roll to prevent any failure and make sure the drone completes its task safely. It also feeds in the angle to Blackbox to track the path during the descent and ascent of the journey.

\subsection{Curve of Ascent}

Although there can be many curves for the ascent, the most efficient in the case of timing is the inclined plane. The inclined plane at an angle of 45 degrees takes the least time and works to reach a point of height $\mathrm{x}$ meters and thus for the ascent, an inclined path to a certain height is calculated to minimize time. The inclined plane is the most efficient of all curves in the ascent, where all the forces of nature are bound to act against the drone. Therefore, an easy and efficient approach is easy to maneuver and maintain in the circumstance as the normal forces either cancel each other or some part of themselves in this curve, while all three coordinates depend on themselves to give more accurate and better coordinates for the drone to move.

The equation for the inclined plane should be (i.e., angle of inclination should be 45 degrees) for a perfect incline plane:

$$
\mathrm{x}=\mathrm{y}=\mathrm{z},
$$

\subsection{Curve of Descent}

The Brachistochrone curve or curve of the fastest descent is used to reach the destination point. It is the most efficient curve in case of descent as it increases the efficiency and decreases the time of flight by a great margin.

The equation to be used in the algorithm is:

$$
\begin{aligned}
& X=a(\theta-\sin \theta), \\
& Y=a(1-\cos \theta),
\end{aligned}
$$

where $a$ is the distance of the centre to an endpoint and $\theta$ is the angle between the path and the angle of the UAVs.

The brachistochrone curve is the fastest descent as with all the forces acting in favour of the drone and for this curve, only a small amount of adjustment and forces by the motors 
are required as all the others are provided by gravity and wind. The curve is free to move on the $z$-axis as the curve has specific angles and to accomplish it accurately, the drone has to have free movement along an axis to overcome any threat or damage in its way. The small step guarantees a higher gradient in altitude than any other curve, which makes it more prone to damage from things like buildings, animals, and stones so an axis free to move will prove to be an optimum solution.

\subsection{Euclidean Distance}

For the aerial transport to follow the path of ascent and descent accurately, the Euclidean distance is used. This function is used to calculate the distance between two different points. The two points (initial and final) denote the centre of the path detected. The two points are given coordinates assigned to them by the GPS module and then the Euclidean distance is calculated by a tracking algorithm that tracks the movement of the $\mathrm{AV}$ and calculates the coordinates and guides the vehicle to the path accurately and keeps checking if it is on track while simultaneously using the GPS module.

All these calculations are done simultaneously with the data being analyzed and used in real-time. These algorithms and calculations are carried out using different modules and chipsets on the flight controller of the UAVs. Figure 1 shows the final path followed by the UAVs as calculated by the algorithm.

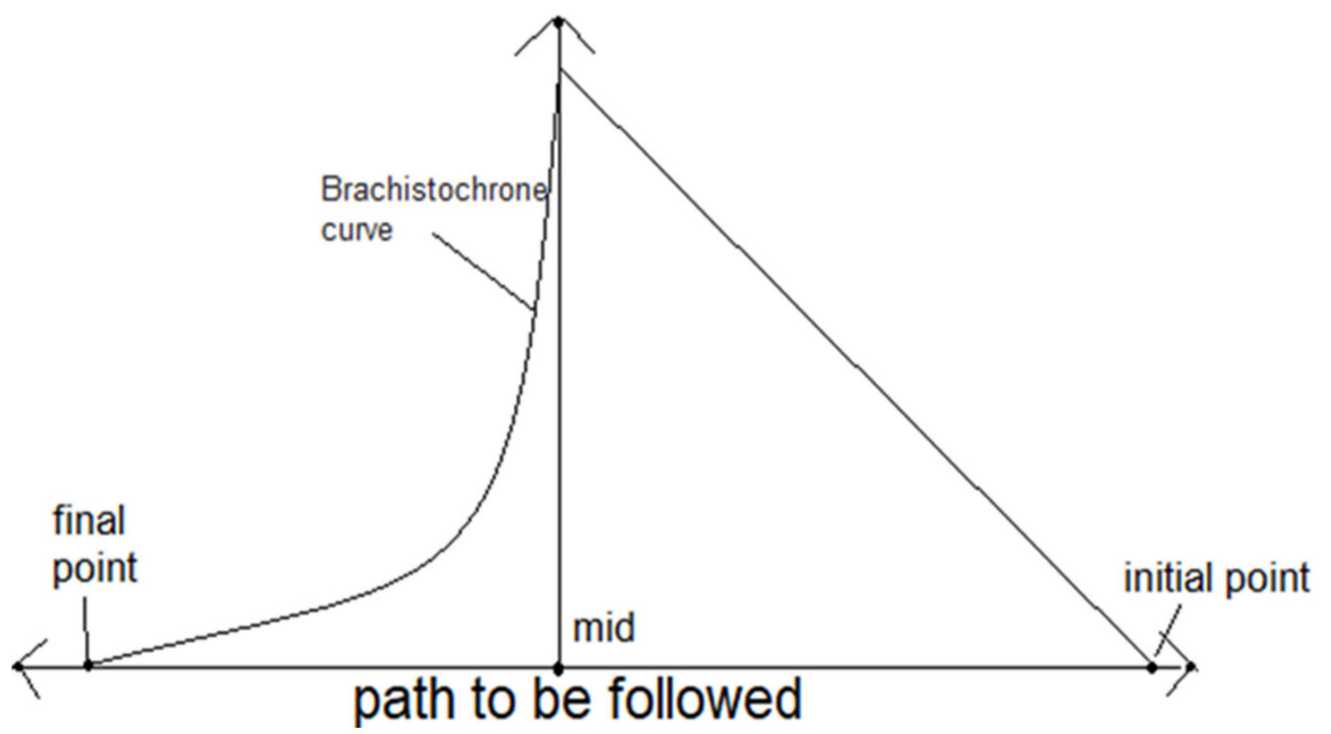

Figure 1. Augmented path.

Now, in this path, we considered only the $\mathrm{x}$ and $\mathrm{y}$-axis as in contradiction to the $3 \mathrm{~d}$ world to keep it simple, but the $\mathrm{z}$-axis is taken as being equal to $\mathrm{x}$ and $\mathrm{y}$ for the ascent as it has to keep a constant angle with the path (45 degrees).

The Euclidean distance interprets all the data in coordinates identifying every object with their coordinates and with its help, the drone will not only have decreased processing time but also by reading the coordinates of an incoming danger or a threat, the drone will also obtain help to identify the threat so it can work out the protocol to avoid it, thus helping in the task at hand and minimizing the chances of failure.

For the descent, the Brachistochrone curve is mainly for the $\mathrm{x}$ and $\mathrm{y}$-axis so the $\mathrm{z}$-axis could be kept constant.

\subsection{GPS (Global Positioning System)}

The GPS is used for the location of the initial and final destination of the journey and also the tracking of the UAVs. The drone has to be tracked through a remote computer and all the data is stored and manipulated by an AI; however, the tracking can be easily done through the GPS while also monitoring the progress and the path of the drone. Additionally, 
in case of failure, the GPS can be tracked to locate the drone for its retrieval. Figure 2 represents the architecture of the proposed augmented path-time model.

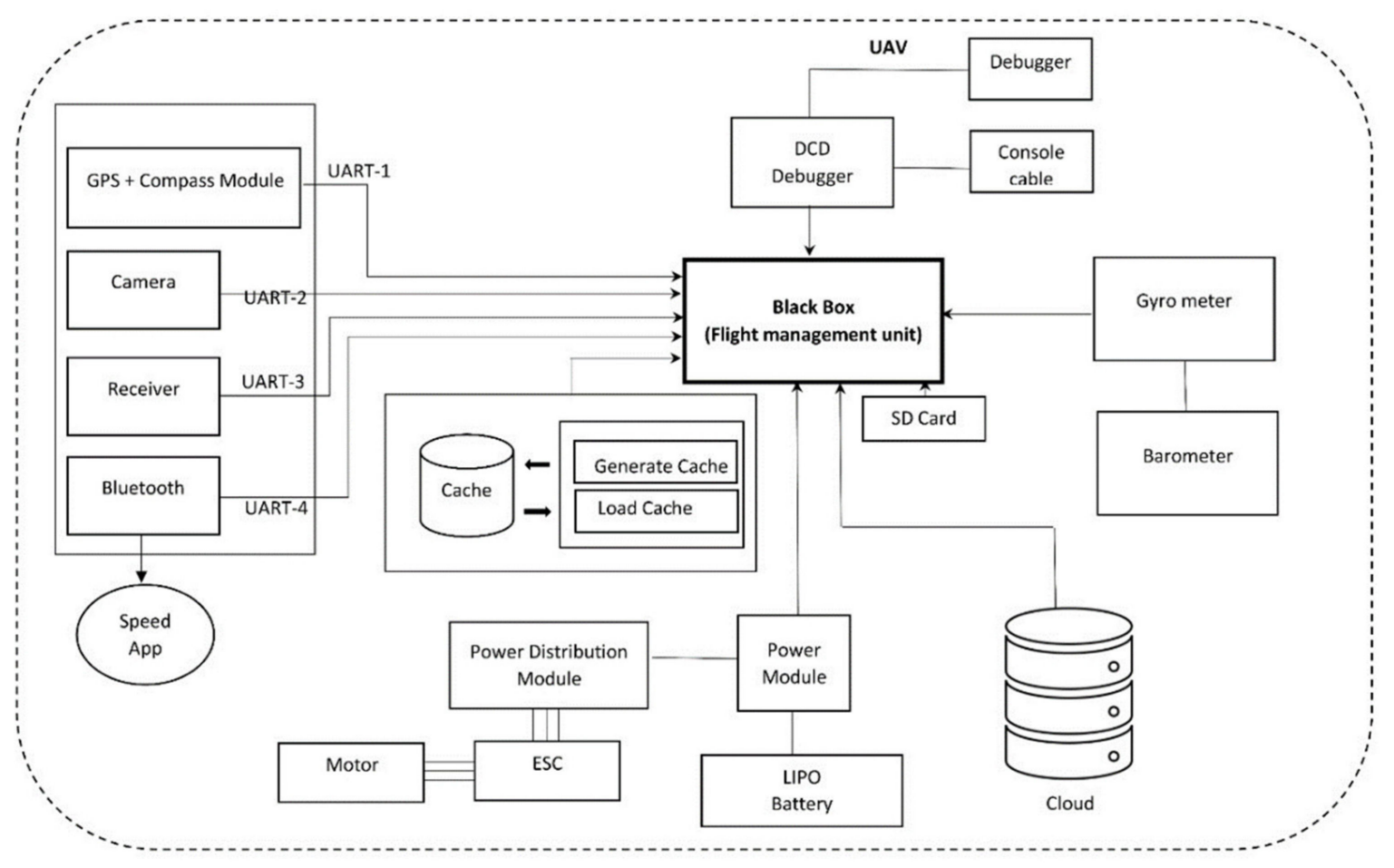

Figure 2. Proposed augmented path-time architecture.

\section{Augmented Path Mapping Algorithm}

Algorithm 1 is the first initializer of the program it sets of the system. It is the trigger, after which all the later algorithms follow. The Euclidean function is initialized. This function is used for calculating the set of values that defines the set of coordinates that the drone has to follow for keeping on the path. It also calculates the midpoint of the path. This midpoint is used for finding the point of descent at which the drone has to decrease its altitude to reach the ground at precisely the exact location.

To keep everything in check and track the values, a counter is set up with an initial value of zero. The counter values decide what is being calculated at the point being calculated.

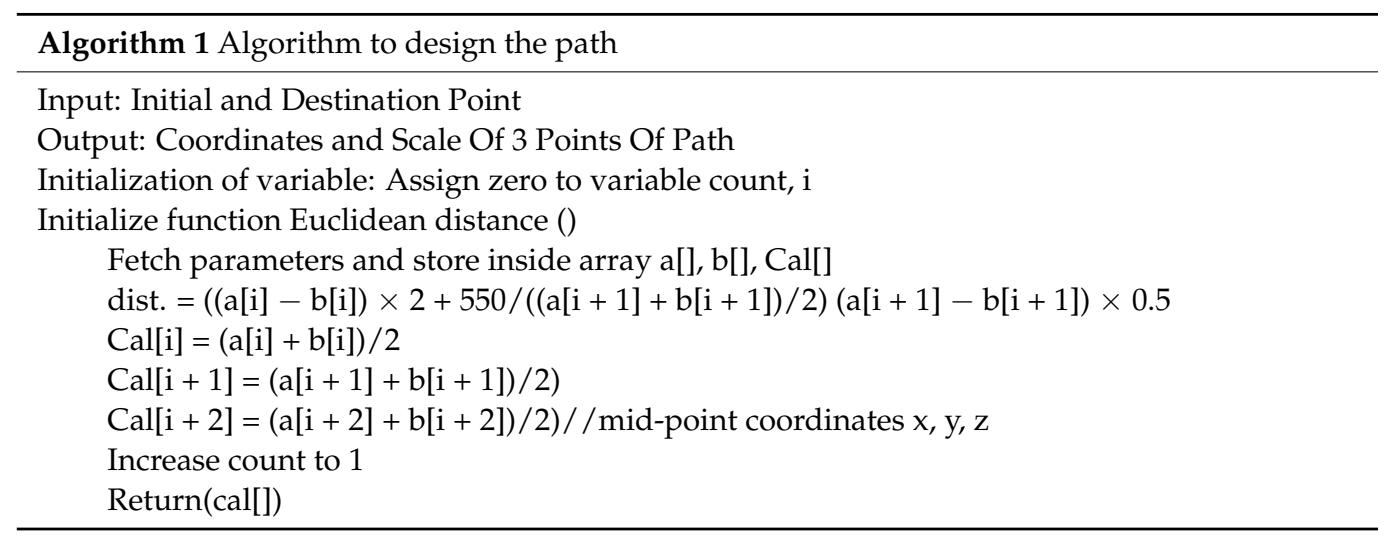

Algorithm 2 is the ascent function that is used to define the path the quad has to follow while starting its ascent. The path is to be defined on all the axis, i.e., $x, y, z$-axis. There is also a mid-variable that keeps checking the coordinates and at precisely the midpoint the function ascent is ended, or the regulated height through the barometer readings to fly below the legally allowed height for the UAVs. 
Now, there are two conditions if the midpoint or the height limit is reached first and that triggers either of the three or four functions, i.e., descent or the straight path.

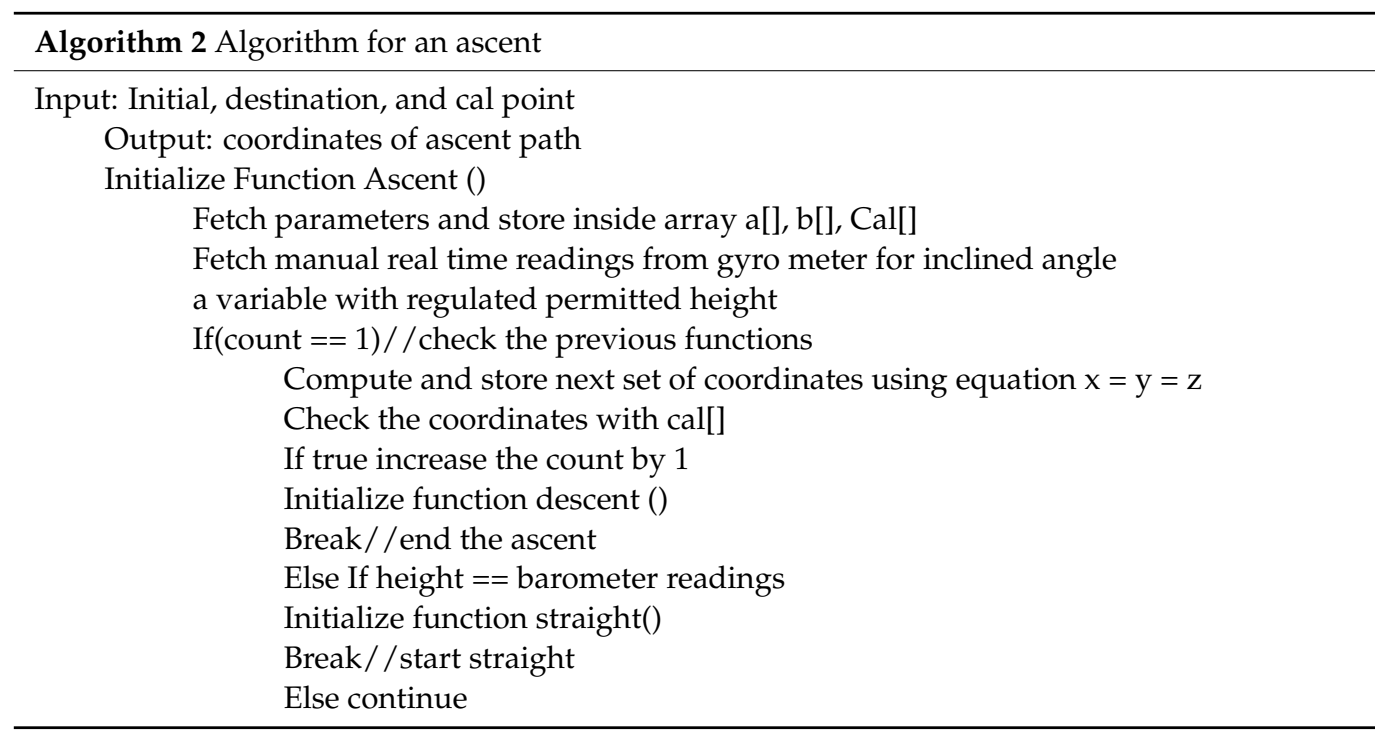

If the mid-point is reached first, then Algorithm 3 or the descent function is initialized. The descent function is used to track the descent path using their respective equations. It has an end variable, which checks the final coordinates with the live coordinates and thus the progress is checked. All this is calculated in real-time and modified simultaneously during the flight of the quad (see Algorithm 3).

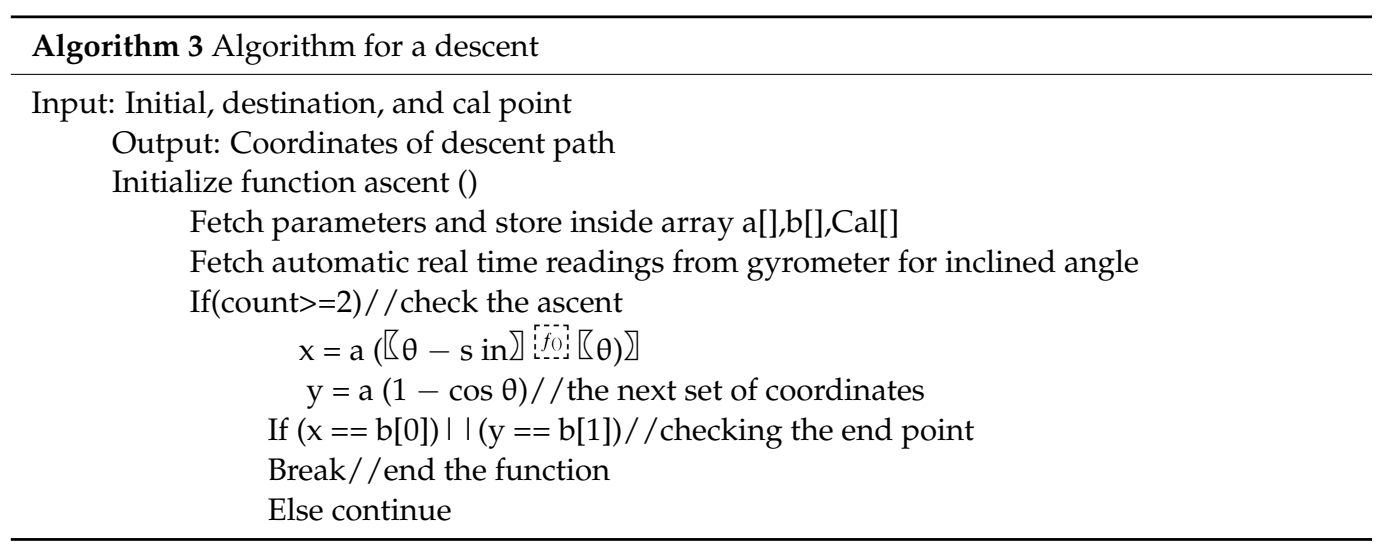

If the height limit is reached first, Algorithm 4 is initialized. The straight function limits the drone movement from further change in the y-axis as the optimum height for the UAVs is reached and as it cannot go above, it moves in a straight line until the midpoint of the whole path is reached.

All the coordinates beside the mid-point are checked in real-time and values are called every instance from the sensors in place. The values are then calculated again for the path. This system is highly efficient as any changes or complications during the flight can be overcome by precautionary measures and then the path is again followed (see Algorithm 4). 


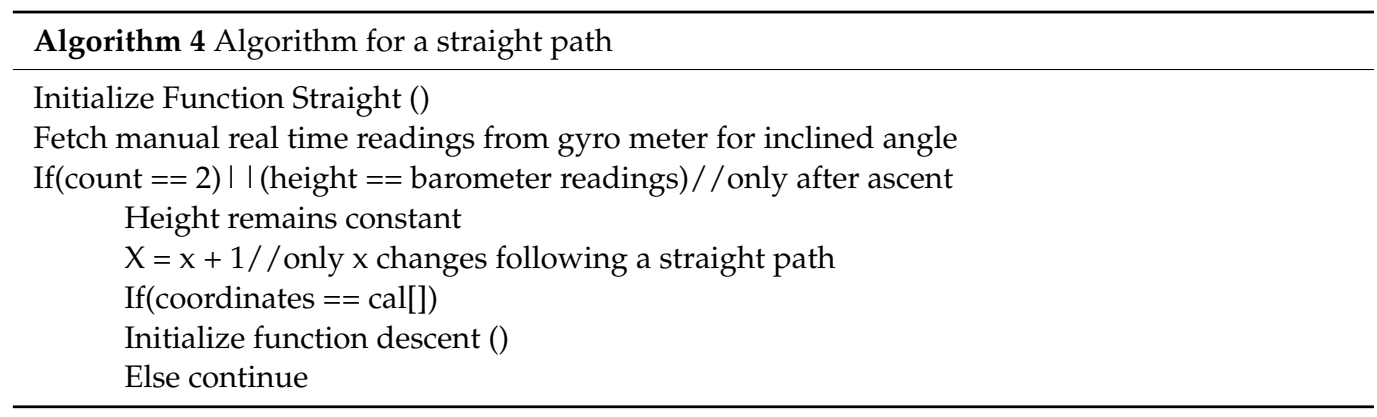

\section{Calling Technique of the Algorithm}

When the algorithm is compiled, it calls for values from the Blackbox via the function call, which already has the simultaneous values of the respective modules, i.e., gyro, barometer, etc., which is used in the flight controller for balancing and motor control of the quad. The Blackbox then provides the values of the respective functions in the algorithm, which gives the next set of coordinates for the drone to move on and this process goes on, thus giving the final path that the drone has to follow for the final destination.

The data is sent through the program at every single instance of time as the coordinates are generated throughout the journey for the progress and smooth running of the drone. All the data is processed for the next coordinates and each coordinate is checked to keep track of the ascent and descent.

Figure 3 is a small representation of the working of the algorithm from input to output.

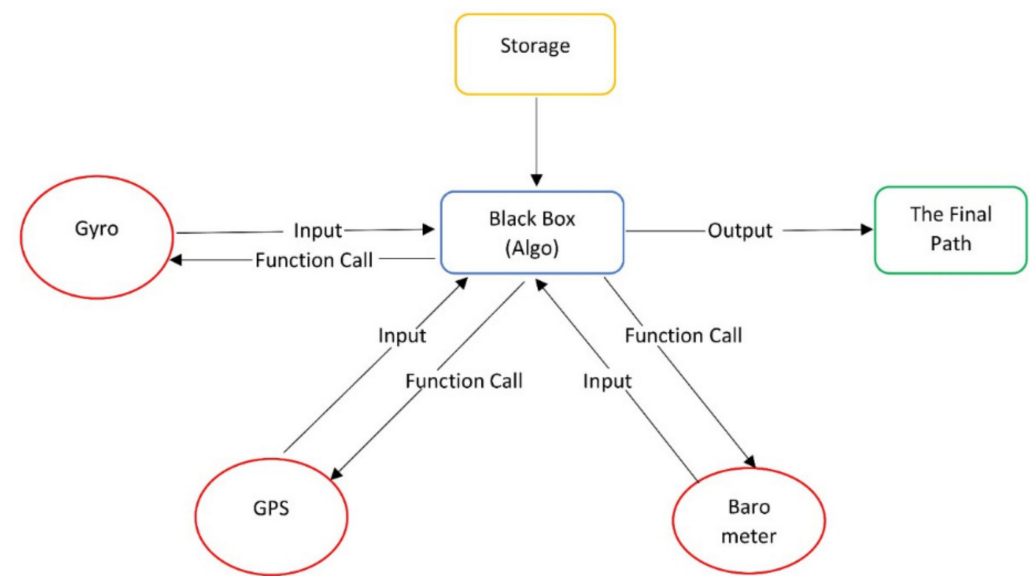

Figure 3. The flow of data.

\section{Experimental Setup}

In Tables 1-3, below, we calculated the time of the original path and after on the modified path. Our research shows that if we can follow the path, the time optimization and thus the output of these UAVs can be increased for better usage of resources and better output. The efficiency is calculated by Equation (4):

$$
\text { Efficiency }=(\text { final time }- \text { initial time }) / \text { initial time } \times 100 \text {, }
$$


Table 1. Time of ascent.

\begin{tabular}{ccccc}
\hline $\begin{array}{c}\boldsymbol{X} \text {-Axis (in } \\
\text { Meters) }\end{array}$ & $\begin{array}{c}\text { Y-Axis (in } \\
\text { Meters) }\end{array}$ & $\begin{array}{c}\text { Original Time } \\
\text { of Ascent (in s) }\end{array}$ & $\begin{array}{c}\text { New Time of } \\
\text { Ascent (in s) }\end{array}$ & $\begin{array}{c}\text { Efficiency of } \\
\text { Ascent }\end{array}$ \\
\hline 1 & 1 & 0.1 & 0.05 & 0.5 \\
10 & 10 & 1 & 0.5 & 0.5 \\
20 & 20 & 2 & 1 & 0.5 \\
50 & 50 & 5 & 2.5 & 0.5 \\
100 & 100 & 10 & 5 & 0.5 \\
200 & 200 & 20 & 10 & 0.5 \\
500 & 500 & 50 & 25 & 0.5 \\
1000 & 1000 & 100 & 50 & 0.5 \\
\hline
\end{tabular}

Table 2. Time of descent.

\begin{tabular}{ccccc}
\hline $\begin{array}{c}X \text {-Axis (in } \\
\text { Meters) }\end{array}$ & $\begin{array}{c}\text { Y-Axis (in } \\
\text { Meters) }\end{array}$ & $\begin{array}{c}\text { Original Time } \\
\text { of Descent (in s) }\end{array}$ & $\begin{array}{c}\text { New Time of } \\
\text { Descent (in s) }\end{array}$ & $\begin{array}{c}\text { Efficiency of } \\
\text { Descent }\end{array}$ \\
\hline 1 & 1 & 0.67 & 0.59 & 0.12 \\
10 & 10 & 6.77 & 5.899 & 0.13 \\
20 & 20 & 13.54 & 11.799 & 0.13 \\
50 & 50 & 33.87 & 29.499 & 0.13 \\
100 & 100 & 67.73 & 58.99 & 0.13 \\
200 & 200 & 135.46 & 117.998 & 0.12 \\
500 & 500 & 338.66 & 294.995 & 0.13 \\
1000 & 1000 & 677.32 & 589.99 & 0.13 \\
\hline
\end{tabular}

Table 3. Total time of journey.

\begin{tabular}{cccc}
\hline $\mathbf{X}$-Axis (in Meters) $\times \mathbf{2}$ & Y-Axis (in Meters) $\times \mathbf{2}$ & $\begin{array}{c}\text { Initial Time of } \\
\text { Journey }\end{array}$ & $\begin{array}{c}\text { New Time of } \\
\text { Journey }\end{array}$ \\
\hline 1 & 1 & 0.77 & 0.64 \\
10 & 10 & 7.77 & 6.399 \\
20 & 20 & 15.54 & 12.799 \\
50 & 50 & 38.54 & 31.999 \\
100 & 100 & 77.54 & 63.99 \\
200 & 200 & 155.46 & 127.998 \\
500 & 500 & 363.66 & 319.995 \\
1000 & 1000 & 777.32 & 639.99 \\
\hline
\end{tabular}

Table 1 and Figure 4 show the difference in the initial ascent time and the improved time (theoretically). The efficiency is constant in all the cases due to the. fact that the conditions and assumptions are kept constant throughout. Although, in reality, the efficiency may somewhat differ due to some exterior constraints like wind speed, turbulence motor speed, etc, and the graph will always show an increasing curve. These results show that there will be an increase in efficiency if the drone moves on an inclined path with constant speed, thus decreasing time and finally the power usage.

Table 2 and Figure 5 prove that the descent time can be decreased drastically. Moreover, regarding the initial and augmented path, both are taken to be free fall, i.e., no power usage. To keep the drone on the specified path, some forces are needed from the motors, but that is cancelled, as in the initial case too. To prevent the drone from losing control during free fall and guaranteeing safety, some thrust is maintained throughout. Thus, the energy is cancelled in both and the time frames can be decreased by the augmented Brachistochrone curve, thus giving more output for the same energy input with increasing efficiency. 


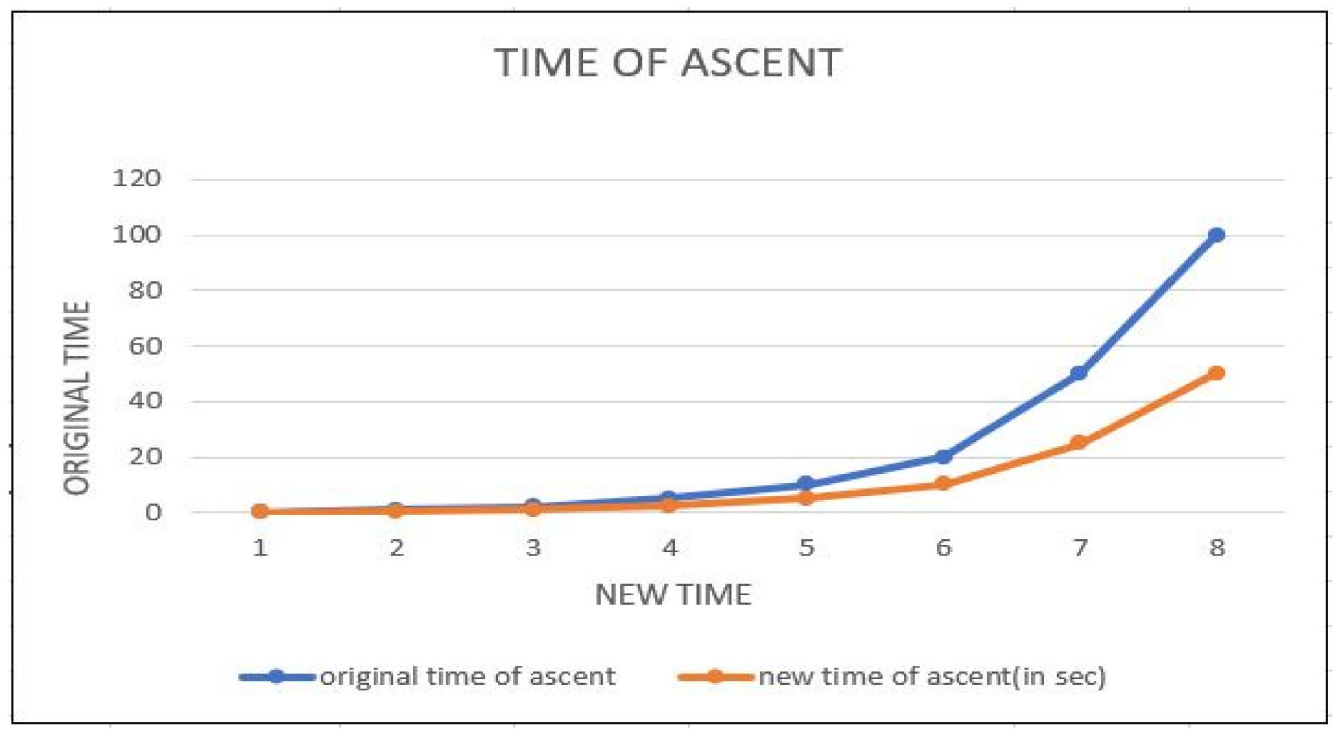

Figure 4. Time of ascent.

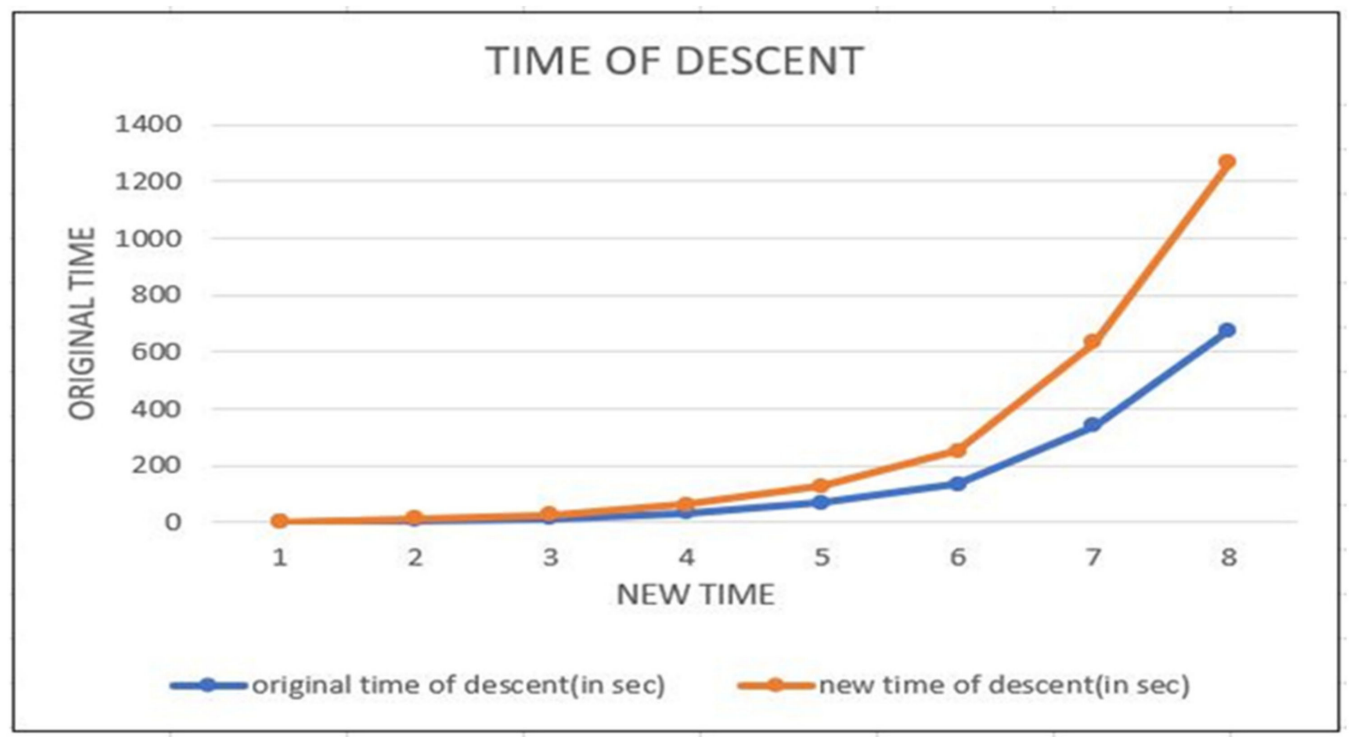

Figure 5. Time of descent.

All the above graphs are made concerning time. As you can see from Figures 5-7, there is a rate of increase in efficiency in both the ascent and descent. Additionally, from the value of Tables 1-3, it is proved that if we apply the above system and include the changes in the present system, the time can be decreased by a significant factor. The graphs show that the traditional approach fails with the medium, i.e., when the medium moves from land to air, a new system is required, which provides a much better result. The graphs also indicate that if we apply for the whole journey, the UAVs have a much higher chance of completing the task than with the traditional approaches as it reduces one of the most limiting factors, i.e., the power factor. With power being used more efficiently, it can cover a greater distance using the same amount of power and thus increase the area of reach and number of tasks at any particular time. 


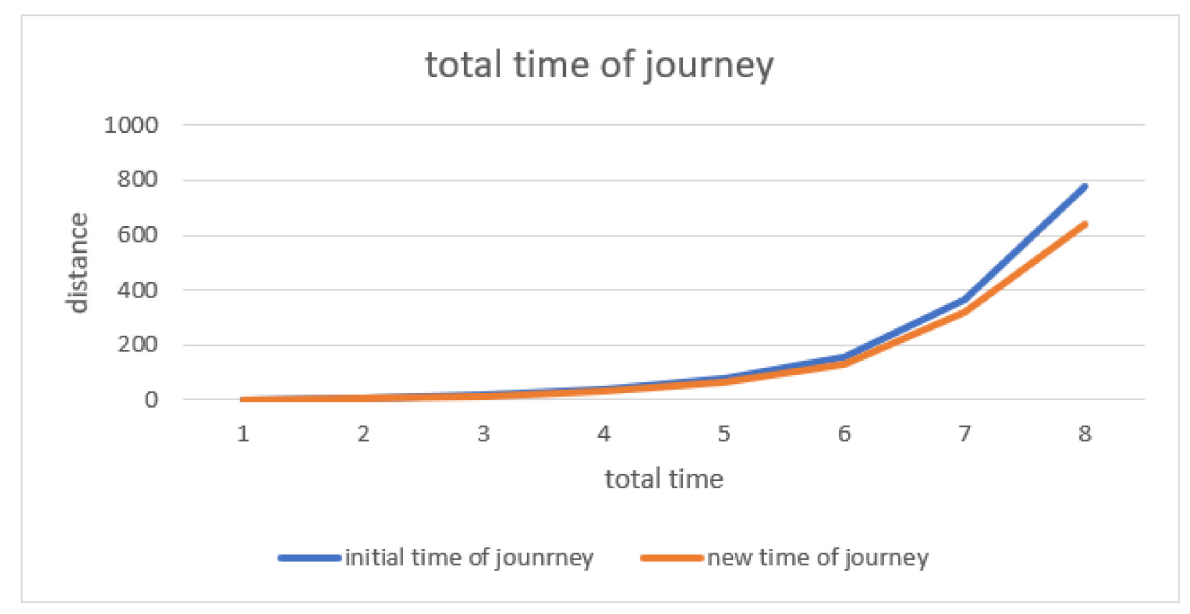

Figure 6. Total time of flight.

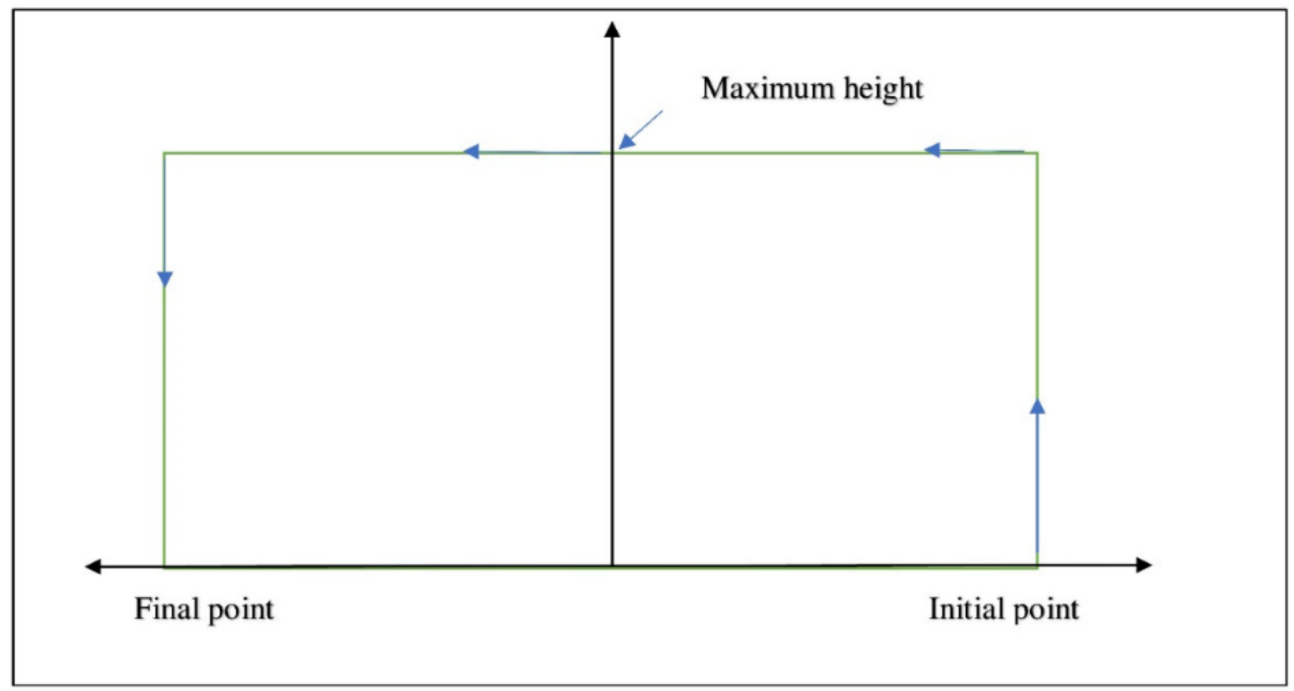

Figure 7. Initial path.

Thus, this indicates that if the above ascent and descent algorithms are used, it provides us with faster drones, which helps in two ways: one is making them more efficient. The ascent algorithm follows an inclined plane, which not only reduces the time but also decreases the path travelled while gaining both distance and height simultaneously. On the other hand, the descent algorithm is a brachistochrone curve and one of the major advantages of this curve is that it can be followed with minimum adjustments and even in a power-saving mode as basically, it uses gravity as its driving force and thus the cumulative efficiency of this path will provide a better overall performance and higher energy output.

For comparison, the path is taken to be a rectangle (2D) or a cuboid shape (3D), where the drone first follows a straight-line path to reach the maximum height and then to reach the destination as shown in Figure 7. It means that first there is a change in the $y$-axis or height only and then after reaching the maximum height, the drone moves forward.

\section{Assumptions}

Ascent

- $\quad$ Speed is constant and due to the presence of external thrust by motors;

- The original path is a rectangle (2d) and cuboid (3d) and the final path is their diagonals, respectively;

- Although the path is changed and calculated at each instance of time, for regularity in the results, it is kept constant and values are taken keeping that in mind. 


\section{Descent}

- In descent, UAV is in a free-fall condition and time is calculated for it;

- No external force is taken into account apart from the forces keeping the drone on the path.

\section{Conclusions and Future Work}

In this paper, there is a way to increase the efficiency of the UAVs with the help of a new algorithm as we see today. The basic measure of these devices is based on distanceefficient systems to reduce their journey; however, as they are in the air, these physical boundaries like roads, plots, etc. disappear. Thus, the advantage of this algorithm is to improve the efficiency by reducing the time and completing tasks faster. This helps in using battery power for a longer period. It brings out more output from these UAVs, thus making them more efficient and increasing their flight duration drastically.

A disadvantage of this algorithm is although it works efficiently, all the axes are fixed, i.e., two axes are fixed for movement while the third is for barrier dodging and so this fixation might be difficult to implement in this AI as some barriers cannot be prevented by just one axis. In this paper, we did not include the internal intelligence transport system, which may be carried out later on [23]. Additionally, the cyber security in our system should be implemented, such that there would not be any possibility for cyber-attacks, which may lead to many problems [24].

For future work, we can bring a more efficient system by using it in real-time applications like on actual UAVs or quads to get real results and more ways to improve. The system could be provided with a third ultrasound or infrared scanning device with an AI, which can easily detect the incoming deviations or barriers, which can prove fatal for the drone and prevent it from using the same algorithm on the third axis while staying on the path. Another major factor is wind speed since the drone has both horizontal and vertical speeds. Wind speed can be detected and used as an upward thrust or downward pull as used by bigger aerial vehicles like aeroplanes, jets, etc. There is also a limit of payload, so if this algorithm is used, it can be used to increase the payload. The real-time application of this idea and algorithm in the real world and also implementing the surrounding environment and speeds in the algorithm is the next step for the improvement of these UAVs as we see today.

Additionally, knowledge of the topological structure of different places where the drone has to work can help to overcome some natural boundaries. The topological map of the place can provide some deep insight about the structure and landmarks of the place and help to escape some like tall grasses or higher altitude land spaces like mountains, thus making the drone moderate its flight accordingly and efficiently.

Although our algorithm gives a perfect ascent and descent time, there might be circumstances or long journeys when these two cannot be followed immediately, thus a straight path is the best option as the quad will not be able to go any further in height and the final destination is still far away so the descent sequence still cannot be initialized. Thus, a way to identify and initiate the specific sequence according to the requirement can be developed that can cover the whole journey irrespective of its length or flight time.

Some precautionary protocols and cyber security should be implemented to prevent harmful and fatal attacks on the system, so that it can be used in the real world.

Author Contributions: Conceptualization, A.K.S.; Methodology, A.K.S., D.A. and M.M.; Supervision, A.Q.M., A.K.S., K.F.T.; Writing an original draft, K.F.T. All authors have read and agreed to the published version of the manuscript.

Funding: This research received no external funding.

Data Availability Statement: The data reported in this study can be found in the sources cited in the reference list.

Conflicts of Interest: The authors declare no conflict of interest. 


\section{References}

1. Jaihyun, L. Optimization of modular drone delivery system. In Proceedings of the 2017 Annual IEEE International Systems Conference, Quebec City, QC, Canada, 24-27 April 2017.

2. Insu, H.; Michael, K.; Murray, A. A commercial drone delivery system for urban areas. IEEE Xplore. In Geocomputation; Springer: Cham, Switzerland, 2017.

3. Miae, K.; Eric, T.; Murray, A. A cost optimization model in multi-agent system routing for drone delivery. In International Conference on Practical Applications of Agents and Multi-Agent Systems; Springer: Cham, Switzerland, 2017.

4. Luigi, D.P.P.; Francesca, G.; Giusy, M. Using Drones for Deliver Process. ISM. 2019. Available online: https://www.sciencedirect. com/science/article/pii/S2351978920305928 (accessed on 27 November 2021).

5. Taner, C. Optimization of battery swapping infrastructure for e-commerce drone delivery. Comput. Commun. 2020, 168, 146-154.

6. Okan, D.; Bahar, Y.K.; Tolga, B. Minimizing energy and cost in range- limited drone deliveries with speed optimization. Transp. Res. Part C Emerg. Technol. 2021, 125, 102985.

7. Courant, R. Calculus of Variables. Global Communications Conference. 1945. Available online: http://www.iri.upc.edu/people/ thomas/Collection/details/57406.html (accessed on 27 November 2021).

8. Yutaka, N. Problem of Quickest Descent. In Artificial Intelligence Applications and Innovations. 1993. Available online: https:/ / www.ijpam.eu/contents/2013-82-3/8/8.pdf (accessed on 27 November 2021).

9. Adams, S.M.; Friedland, C.J. A Survey of Unmanned Aerial Vehicle (Unmanned Aerial Vehicles) Usage for Imagery Collection in Disaster Research and Management. In International Workshop on Remote Sensing for Disaster Response. 2011. Available online: https://www.researchgate.net/profile/Carol-Friedland-2/publication/266465037_A_Survey_of_Unmanned_Aerial_Vehicle_ UAV_Usage_for_Imagery_Collection_in_Disaster_Research_and_Management/links/54d8d9ab0cf24647581c9aa0 / A-Surveyof-Unmanned-Aerial-Vehicle-UAV-Usage-for-Imagery-Collection-in-Disaster-Research-and-Management.pdf (accessed on 27 November 2021).

10. Shashank, A.; Vincent, R.; Sivaraman, A.K.; Balasundaram, A.; Rajesh, M.; Ashokkumar, S. Power Analysis of Household Appliances using IoT. In Proceedings of the 2021 International Conference on System, Computation, Automation, and Networking (ICSCAN), Puducherry, India, 30-31 July 2021; pp. 1-5.

11. Qadir Md, A.; Vijayakumar, V. Combined preference ranking algorithm for comparing an initial ranking of cloud services. Recent Adv. Electr. Electron. Eng. 2020, 13, 260-275. [CrossRef]

12. Nex, F.; Remondino, F. Unmanned Aerial Vehicles for 3D mapping applications: A review. Appl. Geomat. 2014, 6, 1-15. [CrossRef]

13. Zhang, C.; Kovac, J.M. The application of small unmanned aerial systems for precision agriculture: A review. Precis. Agric. 2012, 13, 693-712. [CrossRef]

14. Goodchild, A.; Jordan, T. Delivery by drone: An evaluation of unmanned aerial vehicle technology in reducing $\mathrm{CO}_{2}$ emissions in the delivery service industry. Transp. Res. Part D Transp. Environ. 2018, 61, 58-67. [CrossRef]

15. Laporte, G. The vehicle routing problem: An overview of exact and approximate algorithms. Eur. J. Oper. Res. 1992, 59, 345-358. [CrossRef]

16. Matthew, K.M.; Md, A.Q. An effective way of evaluating trust in intercloud computing. Int. J. Comput. Netw. Inf. Secur. 2017, 9, 36-42.

17. Kothandaraman, D.; Balasundaram, A.; Dhanalakshmi, R.; Sivaraman, A.K.; Ashokkumar, S.; Vincent, R.; Rajesh, M. Energy and Bandwidth Based Link Stability Routing Algorithm for IoT. Comput. Mater. Contin. 2021, 70, 3875-3890. [CrossRef]

18. Murray, C.C.; Chu, A.G. The flying sidekick traveling salesman problem: Optimization of drone-assisted parcel delivery. Transp. Res. Part C Emerg. Technol. 2015, 54, 86-109. [CrossRef]

19. Poikonen, S.; Wang, X.; Golden, B. The vehicle routing problem with drones: Extended models and connections. Networks 2017, 70, 34-43. [CrossRef]

20. Sacramento, D.; Pisinger, D.; Ropke, S. An adaptive large neighborhood search metaheuristic for the vehicle routing problem with drones. Transp. Res. Part C Emerg. Technol. 2019, 102, 289-315. [CrossRef]

21. Roman, R.C.; Precup, R.E.; Petriu, E.M. Hybrid Data-Driven Fuzzy Active Disturbance Rejection Control for Tower Crane Systems. Eur. J. Control 2021, 58, 373-387. [CrossRef]

22. Zhu, Z.; Pan, Y.; Zhou, Q.; Lu, C. Event-Triggered Adaptive Fuzzy Control for Stochastic Nonlinear Systems With Unmeasured States and Unknown Backlash-Like Hysteresis. In IEEE Transactions on Fuzzy Systems; IEEE: Piscataway, NJ, USA, 1 April 2021; Volume 29, pp. 1273-1283.

23. Kosacka-Olejnik, M.; Kostrzewski, M.; Marczewska, M.; Mrówczyńska, B.; Pawlewski, P. How Digital Twin Concept Supports Internal Transport Systems?-Literature Review. Energies 2021, 14, 4919. [CrossRef]

24. Tubis, A.A.; Werbińska-Wojciechowska, S.; Góralczyk, M.; Wróblewski, A.; Ziętek, B. Cyber-Attacks Risk Analysis Method for Different Levels of Automation of Mining Processes in Mines Based on Fuzzy Theory Use. Sensors 2020, 20, 7210. [CrossRef] [PubMed]

25. Kalpana, K.; Paulchamy, B.; Priyadharsini, R.; Sivaraman, A.K.; Vincent, R.; Muralidhar, A.; Tee, K.F. Design and performance analysis of low power high-speed adder and multiplier using MTCMOS in $90 \mathrm{~nm}, 70 \mathrm{~nm}, 25 \mathrm{~nm}$, and $18 \mathrm{~nm}$ regime. In Advances in Parallel Computing (Smart Intelligent Computing and Communication Technology); IOS Press: Amsterdam, The Netherlands, 2021; Volume 38, pp. 409-416. 
26. Md, A.Q.; Vijayakumar, V. Dynamic ranking of cloud services for web-based cloud communities: An efficient algorithm for rating-based discovery and multi-level ranking of cloud services. Int. J. Web-Based Communities 2019, 15, 248-270. [CrossRef]

27. Gayathri, R.; Vincent, R.; Rajesh, M.; Sivaraman, A.K.; Muralidhar, A. Web-acl based dos mitigation solution for cloud. Adv. Math Sci. J. 2020, 9, 5105-5113. [CrossRef]

28. Solomon, M.M. Algorithms for the Vehicle Routing and Scheduling Problems with Time Window Constraints. Oper. Res. 1987, 35, 254-265. [CrossRef] 